\title{
RÉPONSES DES VÉGÉTAUX LIGNEUX MÉDITERRANÉENS À L'ACTION DU FEU'
}

\author{
L. Trabaud*
}

\begin{abstract}
RÉSUMÉ.-On étudie les réponses des végétaux ligneux méditerranéens a l'action du feu dans une zone autour de Montpellier (France). L'auteur suit sur 19 ans (1969-1987) l'évolution de 35 parcelles expérimentales, soumises au feu pendant le printemps ou l'automne et avec différentes fréquences: 2 , 3 et 6 ans. Par ailleurs, étudie 52 zones d'observation brülées par des feux sauvages pendantles dix premieres années après l'incendie. L'auteur conclut en montrant que dans l'ensemble, les végétaux ligneux du bassin méditerranéen résistent aufeu. Toutefois, certains (p. ex. Cistus monspeliensis, Juniperus oxycedrus, Rhamnus alaternus) peuvent être éliminés lorsque les feux sont trop fréquents. En revanche, d'autres ( $p$. ex. Quercus coccifera, $Q$. ilex et $Q$. pubescens) montrent une résistance très forte. La saison des feux a une grande importance sur les capacités de régénération des espèces, et plus particulièrement celles qui sont extremement sensibles à la fréquence
\end{abstract} des incendies.

RESUMEN.- Se estudia la respuesta de diferentes especies leñosas mediterráneas a la acción del fuego en los alrededores de Montpellier (Francia). El autor sigue durante 19 años (1969-1987) la evolución de 35 parcelas experimentales sometidas a fuegos provocados en primavera o en otoño y con distintas frecuencias: 2, 3 y 6 años. Además controla 52 áreas quemadas con fuegos naturales durante los 10 años posteriores al incendio. Concluye señalando que en conjunto las especies leñosas de la cuenca mediterránea son bastante resistentes al fuego. Sin embargo, algunas como Cistus monspeliensis, Juniperus oxycedrus y Rhamnus alaternus llegan a ser eliminadas cuando los fuegos son muy frecuentes. Por el contrario, otras (Quercus coccifera, Q. ilex y Q. pubescens, por ejemplo) muestran una resistencia muy fuerte. La estación en que se quema tiene gran importancia en la capacidad de regeneración de las especies, especialmente en las extremadamente sensibles a los incendios repetidos.

ABSTRACT.- The response of different mediterranean wood species to fire action is studied in the neiglibourhood of Montpellier (France). Along 19 years (1969-1987), the author follows the evolution of 35 experimental plots undergoing artificial fires in the spring and fallow, and with different frequencies: 2, 3 a 6 years. Hé controls moreover 52 areas burned from natural fires, for ten years after the burning. Concluding remarks show that woody species of

1 Recu en février 1992.

- Centre Ecologie Fonctionnelle et Evolutive (C.N.R.S.), route de Mende - B.P. 5051, 34033Montpellier Cédex-France. 


\begin{abstract}
Mediterranean region are, in general, rather fire-resistant. Some of them however, such as Cistus monspeliensis, Juniperus oxycedrus and Ramnus alaternus, con be eliminated if the fires are too frequent. Other, such as Quercus coccifera, Quercus ilex or Quercus pubescens, show, importance for the regeneration capability of the species, mainly for those being very sensitive to frequent fires.
\end{abstract}

Key words: response of woody species to fire, experimental plots, natural fires, mediterranean basin.

Du fait de sa fréquence passée et actuelle, le feu est un facteur important de la dynamique des communautés végétales qui existent autour du Bassin méditerranéen. Divers auteurs (NAVEH, 1974, 1975; Trabaud, 1974, 1980; ARIANOUTSOU, 1984; TERRADAS, 1987) ont, depuis quelques années, abordé ce problème, considérant surtout le devenir des communautés après le traumatisme créé par le feu.

Dans la région méditerranéenne française, l'action de feu a été, surtout, considérée au niveau des communautés: impact à la fois floristique et structurel (Trabaud, 1970, 1977, 1983; TRABAUd \& LePART, 1980, 1981). Une étude plus précise de l'infuence du feu sur les végétaux ligneux, qui constituent les éléments dominants de la végétation méditerranéenne, apportera de nombreux renseignements sur les réponses de ces végétaux et leur croissance post-incendie.

Pour savoir quel est le devenir réel des phytocénoses après un incendie, il est essentiel de suivre leur évolution au cours du temps soit sur des dispositifs expérimentaux contrôlés, soit dans la nature sur des placettes qui ont été parcourues par des feux sauvages dont le date exacte du dernier incendie est parfaitement connue. Les deux approches ont été utilisées.

Pour une analyse fine et une bonne compréhension de l'action du feu sur les végétaux et la végétation, il est nécessaire d'effectuer des expérimentations sur le terrain avec dés mises à feu contrôlées. En effect, seule l'expérimentation réalisée sur des stades de végétaux identifiés avant le feu peut produire des résultats fiablés. L'expérimentation a été conçue pour analyser:

-l'impact sur la végétation de mises à feu répétées selon diverses fréquences: un feu tous les six ans, un feu tous les trois ans, un feu tous les deux ans; afin de vérifier si des feux très fréquents vont modifier les capacités de régénération des végétaux;

-les effets sur la végétation de la date (saison) des mises à feu, afin de déterminer si les conditions saisonnières qui provoquent des variations du comportement phénologique et physiologique des espèces, modifient les réponses des végétaux.

Dans les placettes, établies sur les zones brâlées par des feux sauvages, la repousse des végétaux ligneux, ainsi que leur accroissement au cours du temps, seront suivis afin de connaître les capacités de croissance des espèces. 


\section{Méthodes, dispositif et nature des observations}

\section{1. Étude expérimentale}

L'expérimentation a été en place sur une colline située à $10 \mathrm{~km}$ au nord de Montpellier. Les parcelles expérimentales sont localisées sur le versant sud-ouest de la colline, constituée par un calcaire lacustre de l'Eocène. Le climat de la zone est méditerranéen humide selon la classification d'EMBERGER (1942, 1971). La saison pluvieuse coincide avec la période à faibles températures; l'été généralement chaud et sec. Sur 19 ans (1969-1987), la moyenne annuelle des températures est de $13,2^{\circ} \mathrm{C}$; la moyenne des températures minimales du mois le plus froid (janvier) est $2,4^{\circ} \mathrm{C}$; tandis que la moyenne des températures maximales du mois le plus chaud (juillet) est de $28^{\circ} \mathrm{C}$. La pluviosité moyenne anuelle atteint $191 \mathrm{~mm}$. La communauté végétale est une garrigue de Quercus coccifera* (Cocciferetum Br.-BI. 1924, sous-association brachypodietosum Br.-BI. 1935; BRAUN-BLANQUET et al., 1952). Le choix de cette communauté a été motivé par l'importance des superficies qu'elle couvre dans le sud de la France, et le fait qu'elle est fréquemment parcourue par les incendies.

Le choix des dates de mises à feu a été pris en fonction des stades phénologiques du Q. coccifera:

-mises à feu de printemps, lorsque le démarrage végétatif printanier de Q. coccifera est effectif; les premières pousses de l'année et les nouvelles feuilles sont largement développées, les fleurs sont apparues. Q. coccifera est a son état d'activité photosynthétique maximale;

-mises à feu d'automne, lorsque les rameaux de l'année de $Q$. coccifera sont aoûtés; le végétal, du moins en apparence, semble au repos.

La fréquence des mises a feu est de "un feu tous les six ans" "pour un groupe de parcelles", "un feu tous les trois ans" pour un autre groupe, et "un feu tous les deux ans" pour un troisième groupe. Des parcelles "témoins", non brûlées, complètent le dispositif expérimental.

Cinq répétitions existent pour, chaque traitement combiné saison $x$ fréquence de mises à feu, ce qui donne un total de 25 parcelles élémentaires, réparties selon un plan factoriel au hasard.

La présence de toutes les espèces situées sur chaque parcelle a été relevée régulièrement tous les ans au mois de mai avant les mises à feu de printemps. Cette expérimentation a duré 19 ans (1969-1987).

\subsection{Placettes brûlées par des feux sauvages}

Après reconnaissance sur le terrain des zones brâlées, dont la date d'incendie était parfaitement connue, le choix des stations d'étude a été déterminé parl'homogénéité apparente des différents types de peuplements.

\footnotetext{
* La nomenclature floristique est celle de "Flora Europaea", TUTIN et al., 1964-1980.
} 
L'étude a été effectuée sur des placettes permanentes installées après le passage du feu, et suivies pendantles dix premières années après l'incendie. C'est au cours de cette période que la reconstitution de la végétation est la plus spectaculaire.

Les 52 placettes d'observation étaient situées dans une zone autour de Montpellier, ayant un rayon de $50 \mathrm{~km}$ environ. Elles sont installées dans des communautés végétales représentatives des phytocénoses les plus fréquemment rencontrées dans cette région:

-taillis de Quercus ilex (13 stations);

-garrigues de Quercus coccifera (15 stations);

-pinèdes de Pinus halepensis ( 7 stations);

-garriges de Rosmarinus officinales (6 stations);

-pelouses de Brachypodium sp. pl. (6 stations);

- cistaies de Cistus monspeliensis (3 stations);

-génistaies de Spartium junceum (2 stations).

La placette d'observation consistait en une ligne permanente de $20 \mathrm{~m}$ de long, dont les piquets repères étaient scellés dans le sol. Les mesures étaient effectuées régulièrement tous les ans au printemps. Pour les espèces dominantes, caractérisant chacun des peuplements étudiés ( $Q$. ilex, Q. coccifera, Rosmarinus officinalis, Cistus monspeliensis, Spartium junceum) et dans ces cas seulement, le hauteur des individus était mesurée systématiquement tous les $2 \mathrm{~m}$ le long de la lingne, soit 10 individus. Dans les autres communautés et pour les espèces non-dominantes, de 2 à 10 individus (pris au hasard, puis repérés et toujours les mêmes par la suite), selon le nombre de présents dans une aire de $100 \mathrm{~m}^{2}$, étaient mesurés pour chaque espèce. La moyenne de ces mesures représentait la hauteur moyenne de l'espèce à l'instant $t$ dans la placette étudiée. Sur les figures sont portées les hauteurs moyennes des espèces correspondant à plusieurs placettes ayant le même âge en fonction du temps après le feu.

\section{Resultats et discussion}

\section{1. Étude expérimentale}

Selon les saisons et les fréquences des mises à feu, le degré de présences de certaines espèces a tendance à décroître (certaines espèces même, disparaissent), pour d'autres il reste inchagé, enfin pour une espèces, il y aurait accroissement du nombre de présences (tableau 1). Les résultats présentés ici correspondent aux nombres de présencés des végétaux ligneux, en comparant ceux observés au début de l'expérimentation (1969), avant les mises à feu, et ceux obtenus 19 ans plus tard (1987), à la fin du troisième cycle expérimental complet de six ans.

Tout d'abord, en absence de mises à feu, dans la végétătion non brúlée, certaines modifications dues à la dynamique naturelle de la végétation, apparaissent. Les degrés de présences de Phillyrea angustifolia, P. media, 
REPONSES DES VEGETAUX LIGNEUX

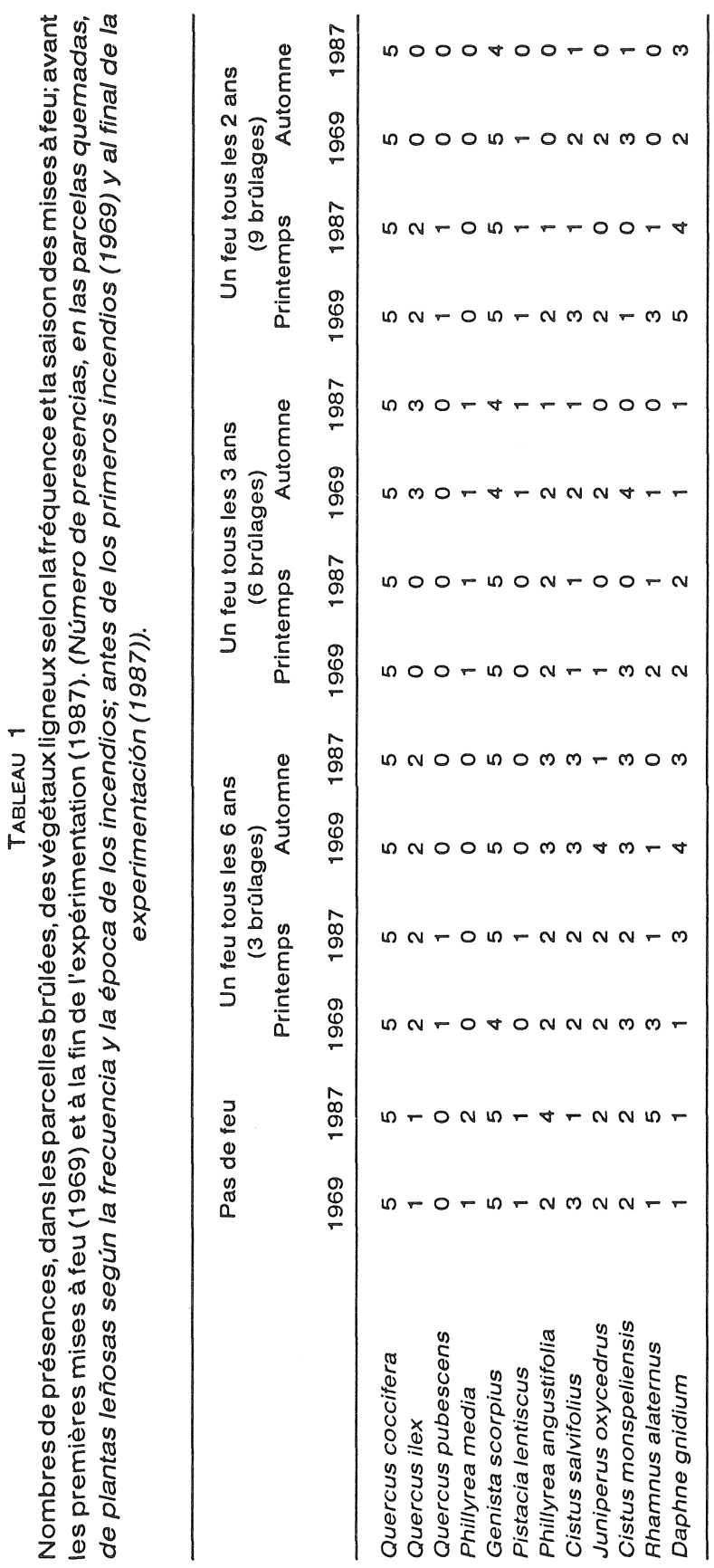

93 
PIRINEOS 140

et Rhamnus alaternus augmentent. Ce changement doit être do aux fruits disséminés par les oiseaux (genre Sylvia; DEBUSSCHE \& ISENMANN, 1983; DEBUSSCHE et al., 1985) qui donnent naissance à de nombreux individus. En outre, la végétation vieillissant devient plus forestière. C'est cette dernière cause qui doit avoir entraîner la disparition de $C$. salvifolius dans certaines parcelles (tableau 1). II n'y a aucun changement pour les autres espèces.

En considération l'action des divers traitements des mises à feu, 4 groupes d'espèces ligneuses ayant des réponses différentes, peuvent être identifiés (tableau 1).

Quercus coccifera, Q. ilex, Q. pubescens, Phillyrea media, constituent un ensemble sur lequel le feu ne semble pas, apparemment, avoir d'effect. Quelles que soient les fréquences et les saisons des mises à feu, ils sont présents à la fin de la période considérée et leur nombre de présences n'a pas changé par rapport à celui d'origine. Ces espèces résistent bien au feu.

Toutefois, du fait de leur trop faible présence dans deux traitements seulement, il est difficile de conclure de façon définitive pour $Q$. pubescens et $P$. media; bien que leur comportement par ailleurs puisse conforter ces résultats.

Genista scorpius, Pistacia lenticus, et Phillyrea angustifolia, forment un groupe légèrement sensible à l'action du feu. Généralement, un feu tous les six ans ne fait pas diminuer le nombre de fois où ils sont présents dans les parcelles. Mais siles fréquences des mises à feu deviennent plus nombreuses (un feu tous les deux ou tous les trois ans), le nombre de présences diminue; cette diminution est d'autant plus marquée que les mises à feu ont été allumées en automne.

Cistus salvifolius, C. monspeliensis, Juniperus oxycedrus, Rhamnus alaternus sont très sensibles à l'action du feu puisque leurs degrés de présences diminuent; ces espèces peuvent même disparaître complèment lorsque la fréquence des mises à feu est élevée (un feu tous les trois ans ou tous les deux ans). L'époque des mises à feu a un effet marqué sur les disparitions, puisque les cas de disparition sont plus nombreux avec les feux d'automne.

Daphne gnidium présente une réponse variable selon la fréquence des mises à feu, mais est la seule espèce ligneuse à réagir favorablement à l'action du feu; son degré de présence diminue dans deux traitements, reste inchangé dans deux autres, et montre un accroissement dans deux autres. $D$. gnidium est une espèce qui résiste bien au feu et caractérise les zones incendiées. La diminution de son degré de présence dans deux traitements proviendrait de la trop grande fréquence des mises à feu. Quant à l'accroissement du degré de présence, il serait dû au mode de dissémination: $D$. gnidium prodiut des fruits charnus qui sont mangés par les oiseaux, puis transportés; une fois installés dans la végétation ouverte par les brâlis, les nouveaux individus ont résisté au passage des feux.

Immédiatement après un feu, tous ces végétaux ligneux, exceptées les deux espèces de Cistus, se régénèrent par voie végétative (rejets); ce sont donc leurs capacités de récupération qui déterminent leur comportement 
(résistance ou disparition) par rapport au feu. En ce qui concerne les deux espèces de Cistus (C. monspeliensis ne se reproduit exclusivement que par vole sexuée après le feu, tandis que $C$. salvifolius a une faible possibilité de régénératon végétative), la sucession fréquente des brâlages (en particulier tous les deux ans) entraîte une impossibilité de reconstitution des individus adultes, les empêchant d'arriver à la floraison et à la fructification, ce qui provoque progressivement un épuisement total de stock de graines situées dans le sol, et donc une élimination de l'espèce (Trabaud, 1980).

\subsection{Placettes brûlées par des feux sauvages}

Après incendie, l'accroissement en hauteur des végétaux ligneux dépend de: (1) leur taille normale caractéristique de l'état adulte (arbres, arbustes, buissons); (2) de leur âge ou de leur "vitalité"; (3) des communautés dans lesquelles ils vivent. Quels sont donc les modes de croissance des espèces observées?

Quercus ilex (fig. 1) est l'espèce dominante caractérisant les taillis qu'elle constitue. Arbre à l'état adulte, elle tend, après le feu, à reprendre un port arboré. Dans les formations forestières (fig. 1a), il faut distinguer la croissance des rejets issus d'arbres considérés comme "jeunes", en fait ayant un diamètre à hauteur de poitrine égal ou inférieur à $10 \mathrm{~cm}$ (représentés par la courbe de croissance la plus rapide), des arbres considérés comme "vieux", ayant des troncs dont le diamètre ètait supérieur à $15 \mathrm{~cm}$. La question d'åge est relative car il n'a pas été possible d'effectuer des sondages dans les troncs; mais par le biais des diamètres, il est possible de considérer que des arbres ayant des grosseurs de diamètres si diffèrentes possédaient des áges différents. Ces aptitudes de croissance plus ou moins rapide selon l'âge, ou l'épaisseur des troncs, a déjà aussi été constaté ailleurs. Ainsi, en Ảmérique du Nord, ROTH et SLEETH (1939) observèrent que la capacité de Quercus a rejeter diminuait en fonction du vieillissement des arbres brâlés. FoWELs (1965) trouva que les réponses de Fagus grandifolia étaient d'autant plus faibles que le diamètre des troncs des arbres brûlés atteignait, ou dépassait, $10 \mathrm{~cm}$. Ces constatations ont été confirmées aussi à partir de Quercus de California (GRIFFIN, 1980; PLUMB, 1980) chez lesquels la vigueur des repousses décroît avec l'âge, et où les arbres brûlés ayant des troncs de faible diamètre présentaient des rejets plus gros que les arbres ayant d'épais diamètres.

Dans les formations nos forestières, la croissance des repousses de Q. ilex est moins rapide (fig. 1b), dépendant des types de communautés. Elle est particulièrement lente chez les individus qui ont été rencontrés dans des pelouses.

Ces différences de croissance seraient dues soit au phénomène de compétition pour la lumière dans les formations forestières, entraînant une poussée des tiges vers le haut, soit au fait que les formations des garrigues sont généralement plus basses, mais aussi qu'elles ont été plus souvent parcourues par les incendies et donc les individus qui les composent présentent un port moins élevé, plus rabougri, qu'ailleurs. 

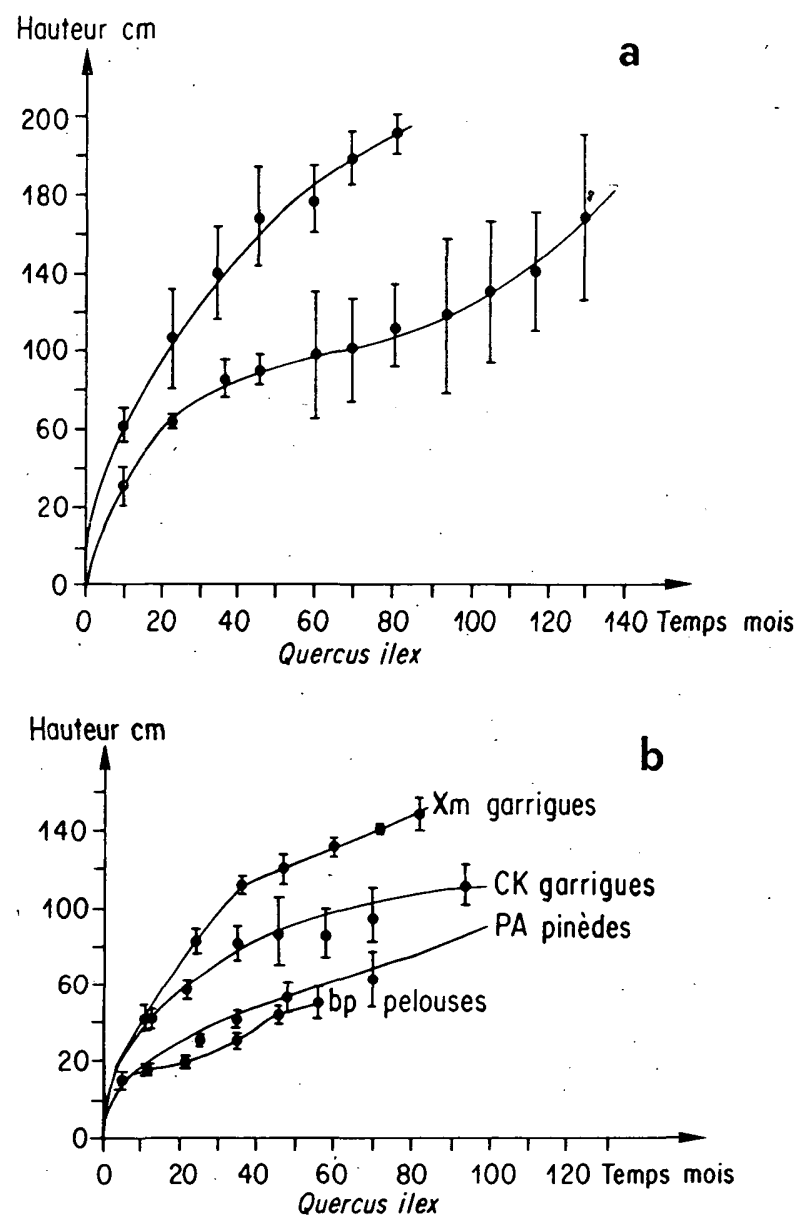

Fig.1. Courbes de croissances des repousses de $Q$. llex après incendie. (a) repousses dans les taillis: courbe supérieure correspondant aux repousses provenant des "jeunes" arbres (diamètres du tron $\leq 10 \mathrm{~cm}$; courbe inférieure correspondant aux repousses provenant des "vieux" arbres (diamètres du tronc $>15 \mathrm{~cm}$ ). (b) repousses dans les autres phytocénoses étudiées. Les sigles correspondent aux abréviations françaises des espèces dominantes des communautés: PA: pinèdes de Pinus halepensis; RO: garrigues de Rosmarinus officinalis; $\mathrm{SJ}$ : genistaies de Spartium junceum; Xm: cistaies de Cistus monspeliensis; bp: pelouses de Brachypodium sp. pl. Les valeurs des intervalles de confiance correspondent au seuil de $5 \%$. Un point sans intervalles de confiance représente les données d'un seul relevé. (Curvas de crecimientos de los rebrotes de Q. ilex tras el incendio. (a) respuestas en el bosque: la curva superior corresponde a los rebrotes procedentes de los árboles "jóvenes" (diámetros del tronco $\leq 10 \mathrm{~cm}$.); la curva inferior corresponde a rebrotes en árboles "viejos" (diámetros del tronco >15 cm.). (b) Rebrotes en las restantes fitocenosis estudiadas. Las siglas corresponden a las abreviaturas francesas de las especies dominantes de las comunidades: PA:-pinares de Pinus halepensis; RO: garrigas de Rosmarinus officinalis; SJ: aliagares de Spartium junceum; Xm: jarales de Cistus monpeliensis; bp: pastizales deBrachypodium sp. pl. Los valores de los intervalos de confianza corresponden al umbral de 5\%. Un punto sin intervalos de confianza representa los datos de un solo muestreo). 
Phillyrea media (fig. 2) présente aussi ce démorphisme de croissance dû probablement aux mêmes causes que pour $Q$. ilex.

Arbutus unedo (fig. 2), espèce rencontrée seulement dans les taillis de Q. ilex, présente deux modes de croissance; l'une extrêmement rapide du fait de certains individus (plus de $2 \mathrm{~m}$ cinq ans après un feu), alors que d'autres croissent lentement. Les individus à croissance rapide vivaient préférentiellement dans les taillis "jeunes" (en compagnie des "jeunes" Q. ilex), tandis que ceux à croissance lente existeraient plutôt dans les "vieux" taillis.

Quercus pubescens (fig. 3), rencontré exclusivement dans les taillis, tend à reprendre très rapidement un port arboré (prés de $2 \mathrm{~m}$ cinq and après un incendie). Lors de l'étude il n'a été observé que dans les jeunes taillis.

Spartium junceum (fig. 3) présente deux modes de croissance dont la plus rapide est celle des individus vivant dans des formations forestières ou les génistaies, chez lesquelles les individus atteignent normalement de grandes tailles.

Quercus coccifera (fig. 4), dans les garrigues où elle est dominante et qu'elle caractérise, cette espèce ligneuse de port buissonnant présente deux modes de croissance selon que les individus avant lincendie possédaient une taille élevée ou pas; les individus hauts produisent de grands rejets, tandis que les individus bas donnent des rejets de taille plus faible (fig. 4a).

Dans les communautés où $Q$. coccifera n'est pas dominant (fig. $4 b$ ), le même phénomène que pour $Q$. ilex apparaît, dû soit à la compétition pour la lumière (croissance plus rapide), soit à la fréquence des incendies ou du port plus bas des autres ligneux dans des formations de garrigues (croissance plus lente).

Phillyrea angustifolia (fig. 5), montre une croissance plus faible lorsqu'il vit dans les pelouses.

Rhamnus alaternus (fig. 5), les individus hauts ayant une croissance rapide sont rencontrés plus fréquemment dans les taillis "jeunes" et les pinèdes, tandis que les individus bas sont plus fréquents dans les "vieux" taillis, les garrigues de $Q$. coccifera, de $R$. officinalis et les pelouses de Brachypodium sp. pl.

Daphne gnidium (fig. 5), dans ce cas encore, les individus ayant une croissance rapide vivent dans les taillis "jeunes". Toutefois, il n'y pas de tendance nette pour les individus viviant dans les différentes autres communautés. Fait à remarquer, c'est la seule espèce qui croît très rapidement au cours des toutes premières années après un incendie: en deux ans, elle atteint une hauteur de $70 \mathrm{~cm}$.

Juniperus oxycedrus (fig. 5) quelles que soient les communautés dans lesquelles vit cette espèce les individus ont la même vitesse de croissance: il n'a pas été possible de les différencier.

II en est de même pour Cistus salvifolius, Buxus sempervirens, Erica multiflora, Rosmarinus officinalis (fig. 6) les individus vivant dans des communautés différentes présentent pourtant les mêmes modes de croissance. 

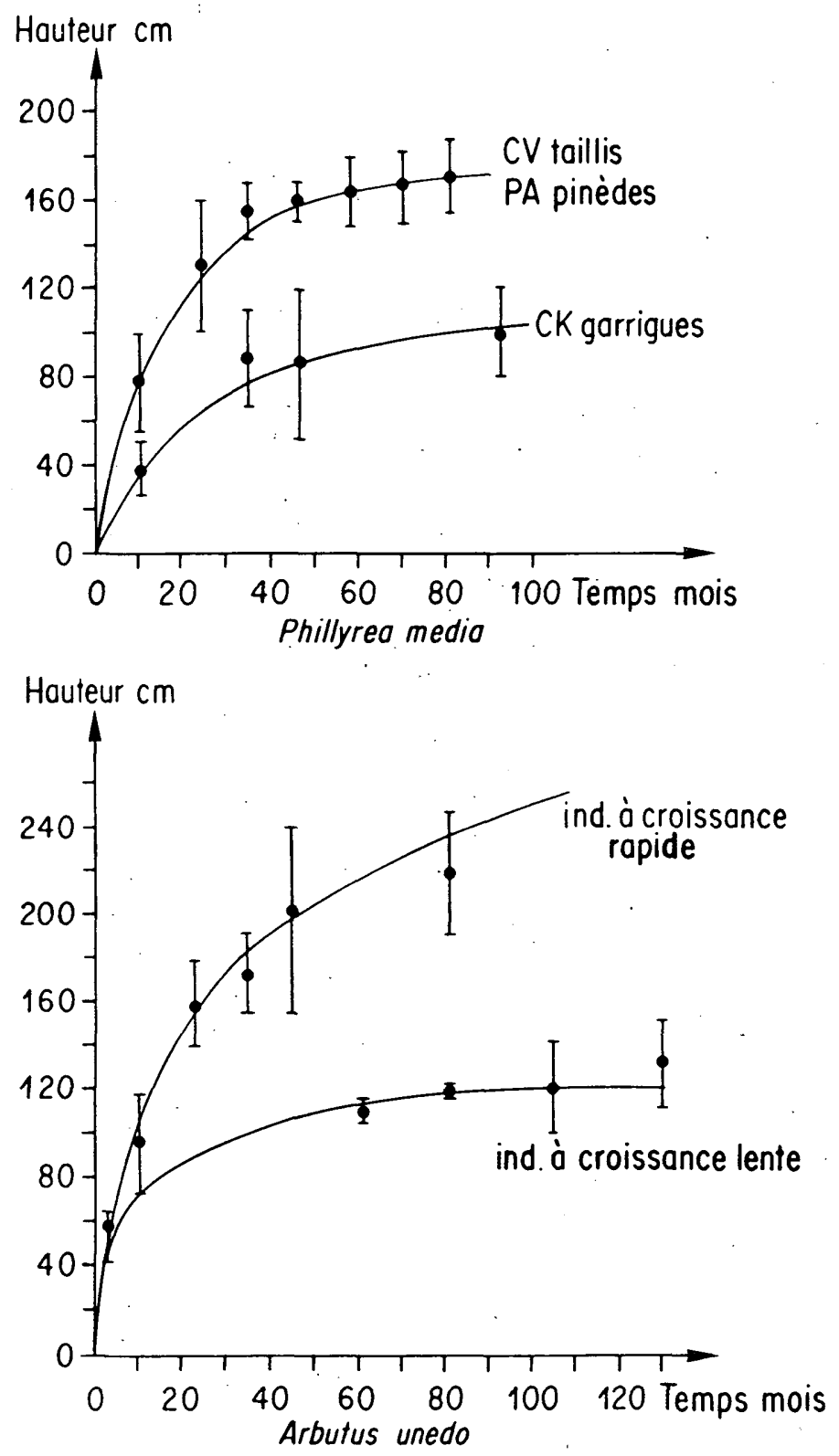

Fig. 2. Courbes de croissances des repousses après incendie. Même légendé que pour la figure 1. (Curvas de crecimientos de los rebrotes tras incendio. Misma leyenda que para la fig. 1).

98 

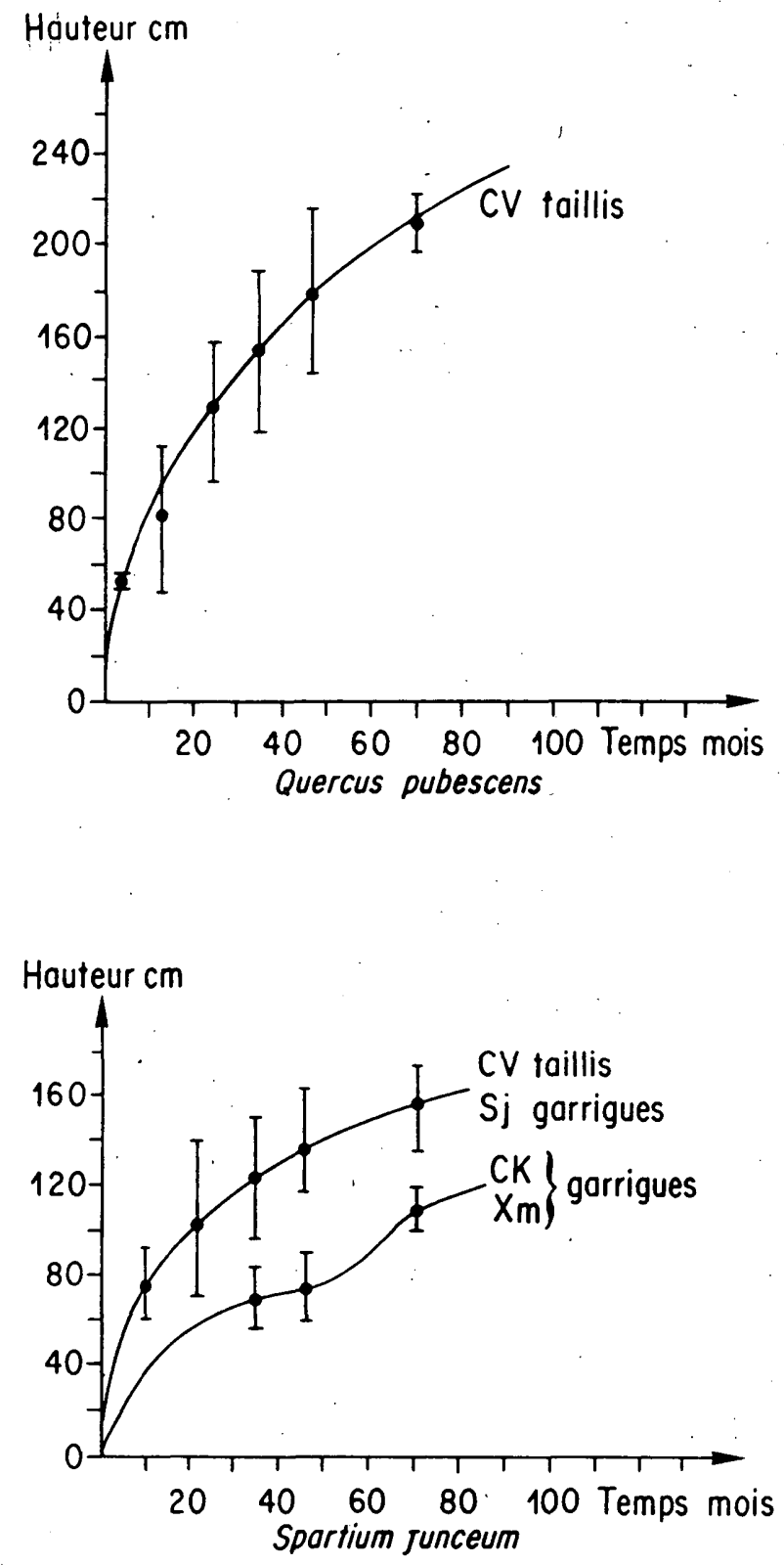

Fig. 3. Courbes de croissance des repousses après incendie. Même légende que pour la figure 1. (Curvas de crecimientos de los rebrotes tras incendio. Misma leyenda que para la fig. 1). 
Hauteur $\mathrm{cm}$

a
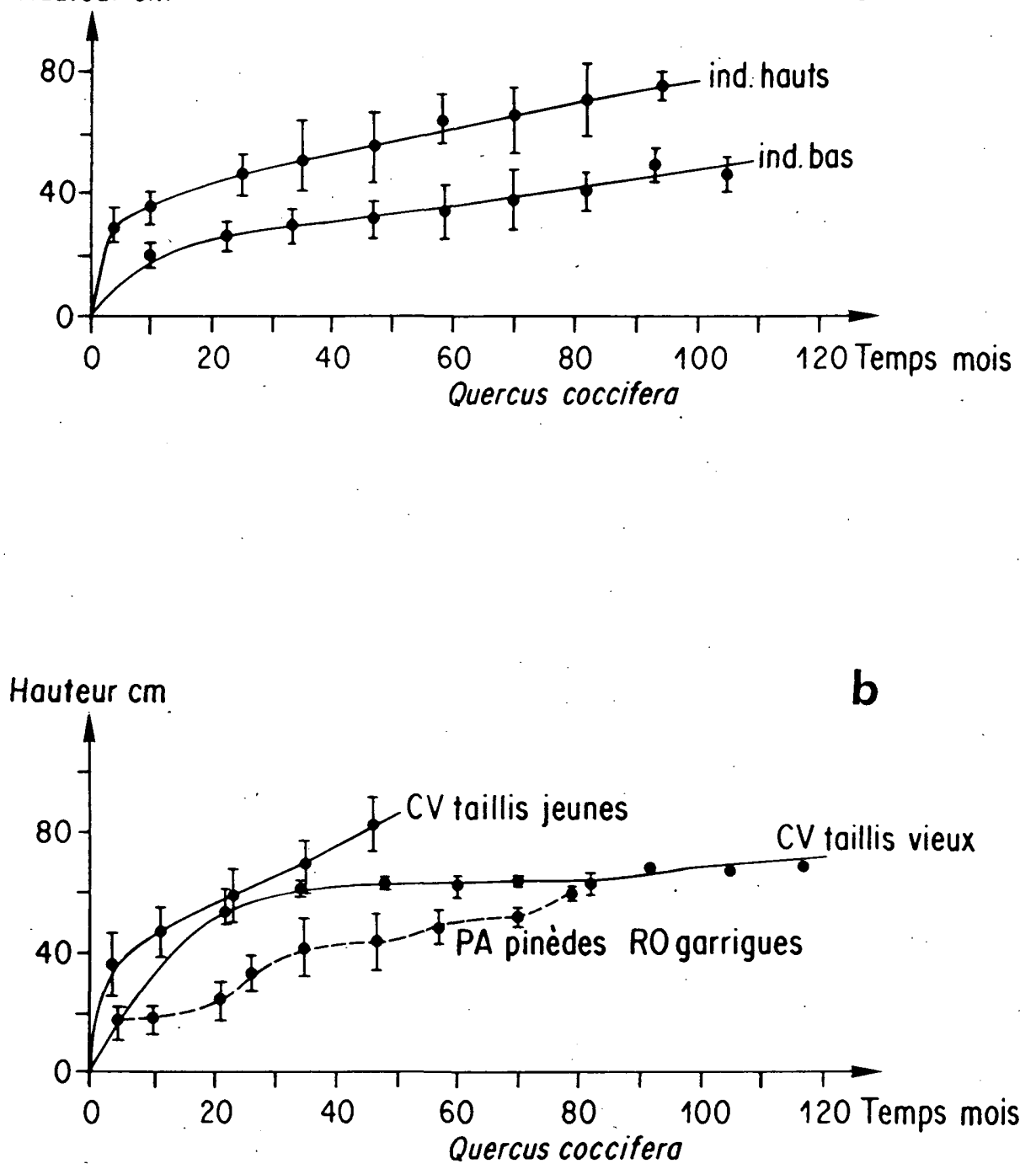

Fig. 4. Courbes de croissance des repousses de $Q$. coccifera après incendie. (a) repousses dans les garrigues formées par l'espèce. (b) repousses dans les autres phytocénoses étudiées. Même légende que pour la figure 1. (Curvas de crecimientos de los rebrotes de Q. coccifera tras incendio. (a) Rebrotes en las garrigas formadas por la especie. (b) Rebrotes en las restantes fitocenosis estudiadas. Misma leyenda que para la fig. 1).

100 
REPONSES DES VEGETAUX LIGNEUX

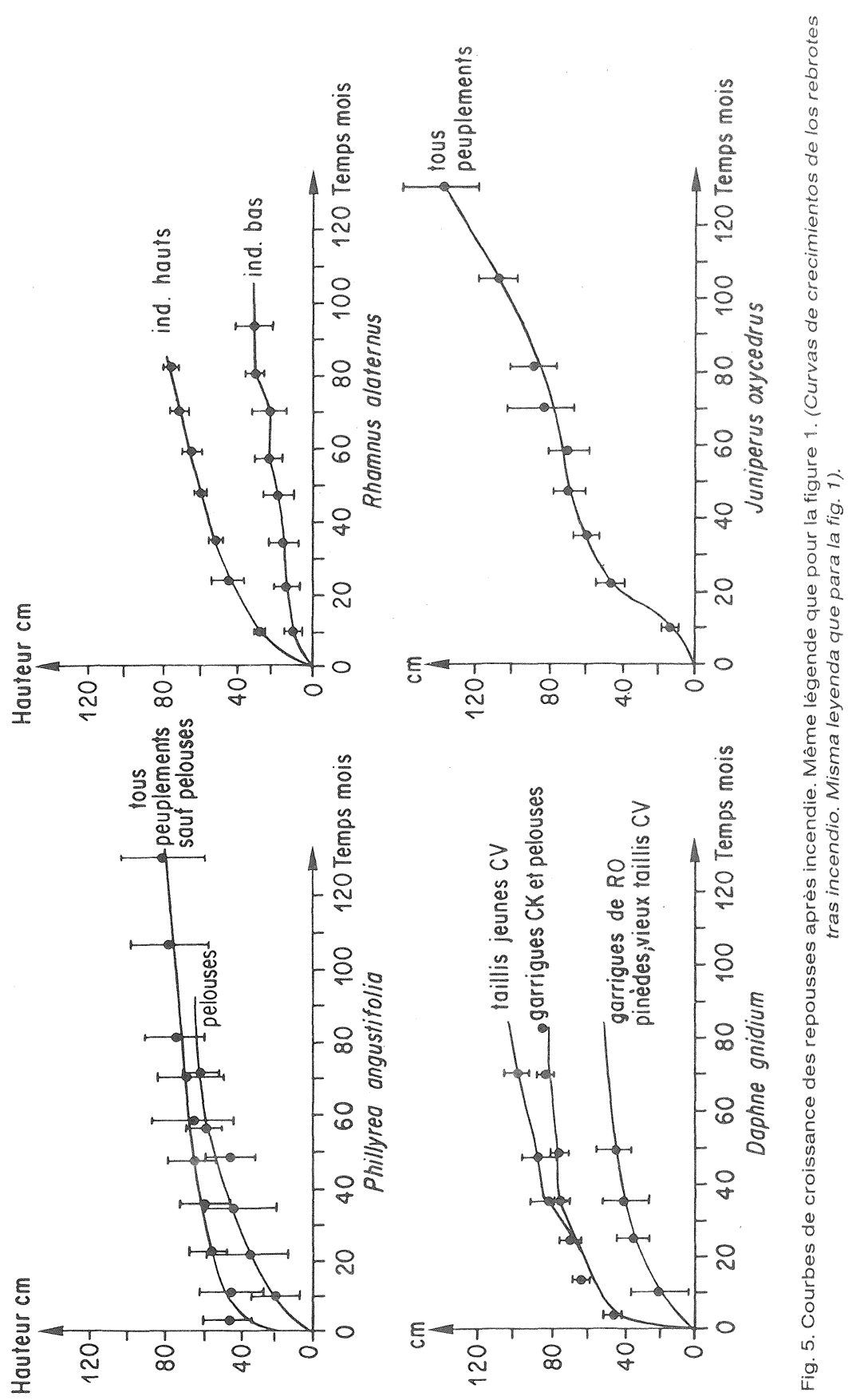

101 
PIRINEOS 140
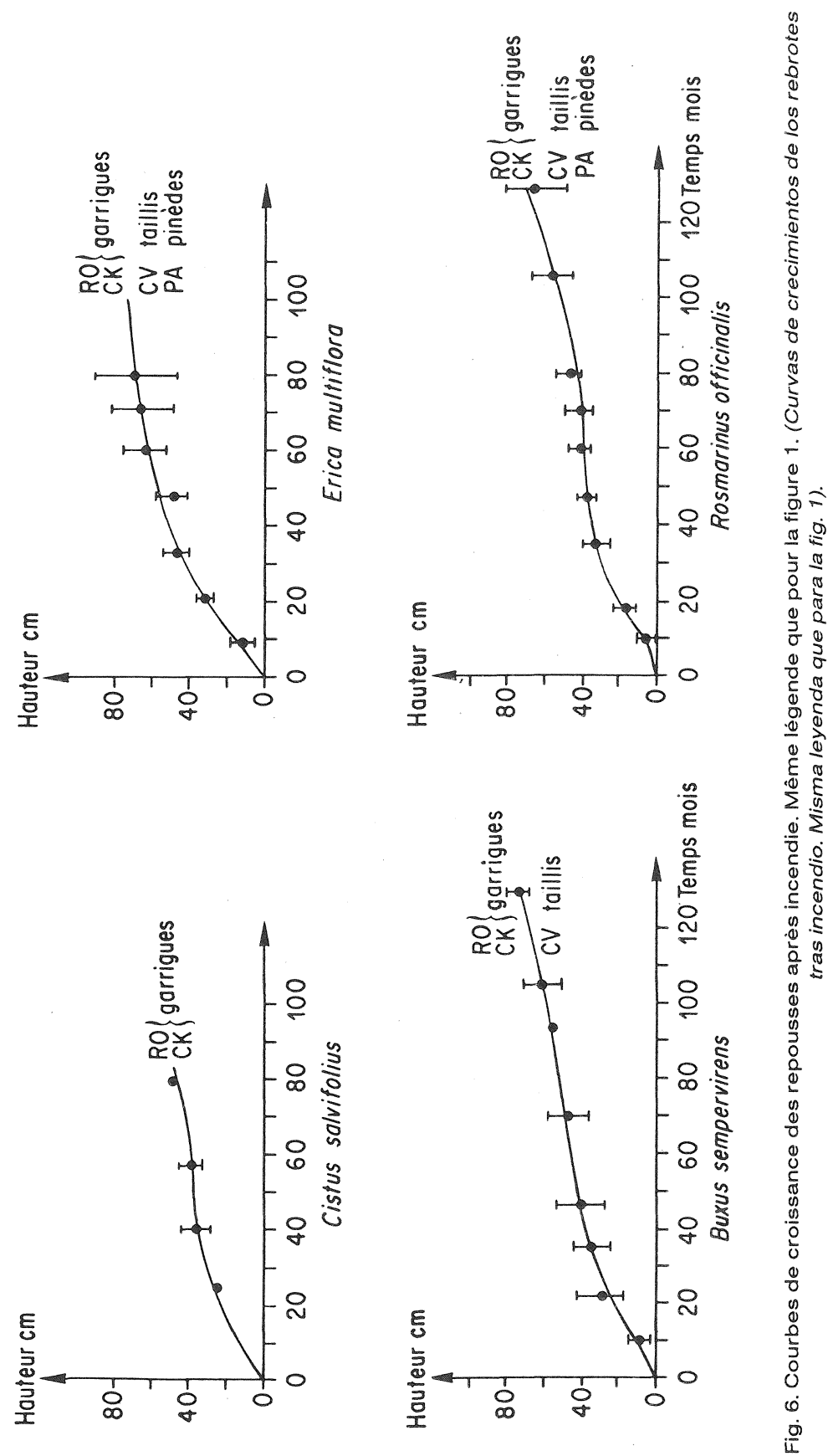
Par contre, Pistacia terebinthus, P. lentiscus, Rhamnus infectoria, Cistus albidus, ont des modes de croissance différents selon les phytocénoses dans lesquelles elles vivent (fig. 7). Généralément, c'est dans les formations forestières (taillis) que la croissance est la plus rapide. Il en est de même pour C. monspeliensis (fig. 8).

En ce qui concerne les cistus, il faut remarquer que $C$. albidus et C. monspeliensisprésent une croissance régulièrement linéaire et indentique, dans les formations de garrigues. $C$. salvifolius ne suit pas cette tendance, et a toujours une croissance plus faible; cela doit être dô au port caractéristique plus prostré de l'espèce dans la région.

La croissance de Pinus halepensis n'est pas présentée dans cet article car elle a déjà fait l'objet d'une précédent étude (TRABAud et al., 1985).

\section{Conclusions}

Dansl'ensemble, les végétaux ligneux du Bassin méditerranéen résistent au feu. Toutefois, certains (p. ex. Cistus monspeliensis, Juniperus oxycedrus, Rhamnus alaternus) arrivent a être éliminés lorsque les feux sont trop fréquents. En revanche, d'autres ( $p$. ex. Quercus coccifera, $Q$. ilex, $Q$. pubescens) montrent une résistance très forte. La saison des mises à feu a une importance sur les capacités de régénération des espèces, et plus particulièrement celles qui sont extrêmement sensibles à la fréquence des incendies: les feux tardifs (ou d'automne) provoquent une diminution de la présence des espèces, voire même entrainent une disparition totale des individus. Bien qu'apparemment, ce sont les espèces qui ne se reproduisent que par voie sexuée après le feu qui semblent les plus touchées, ce sont les capacités de récupération, intrinsèques à chaque espèce, qui déterminent leur réponse (plus au moins grande résistance) au feu, puisque parmi les végétaux se régénèrant végétativement tous ne présentent pas les mêmes aptitudes (ou vitalité) à se maintenir.

Après le passage de l'inciendie, les végétaux ligneux réapparaissent et tendent, au cours du temps, à reprendre le port qu'ils possèdent en absence de traumatisme. Les arbres sont tendre à reconstituer un port arboré, tandis que les buissons vont récupérer leur port buissonnant. L’âge, la taille et la vitalité des individus présents avant le feu sont des facteurs qui déterminent la croissance des repousses après l'incendie.

Pour Q. ilex, les résultats de la présente étude sont comparables à ceux qui avaient été déjà constatés en Amérique du Nord (ROTH et SLEETH, 1939; FoWELLS, 1965; GRIFFIN, 1980; PLUMB, 1980): généralement, la capacité à rejeter diminue avec l'âge, de même les rejets sont plus hauts lorsque les parents brûlés ont des troncs de plus faible diamètre (c'est-à-dire seraient plus "jeunes").

Les vitesses de croissance sont comparables à celles qui ont été observées. Ainsi, parmi les six espèces communes avec le travail de MANSANET TERol (1987): Cistus albidus, Daphne gnidium, Juniperus oxycedrus, 
PIRINEOS 140
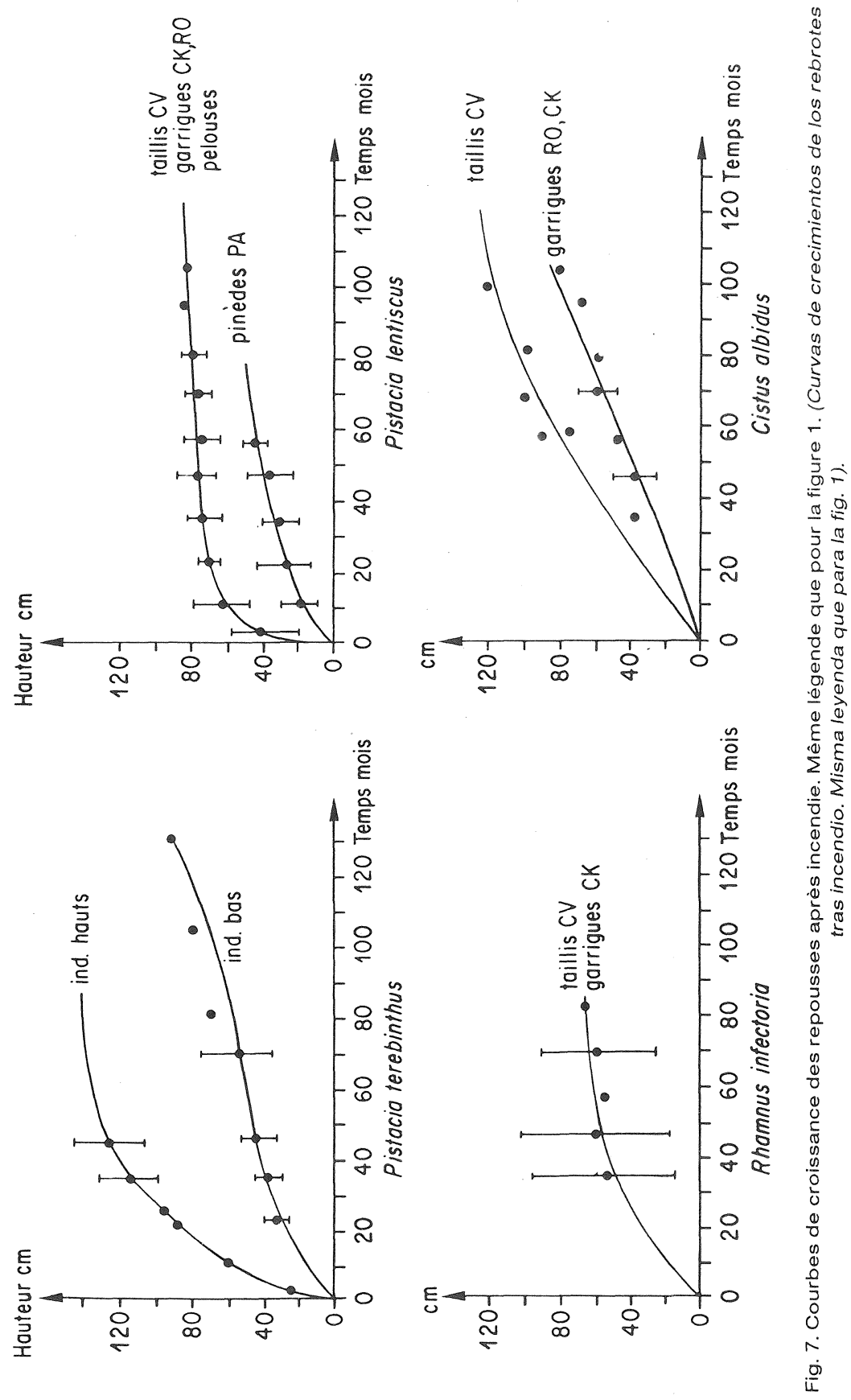


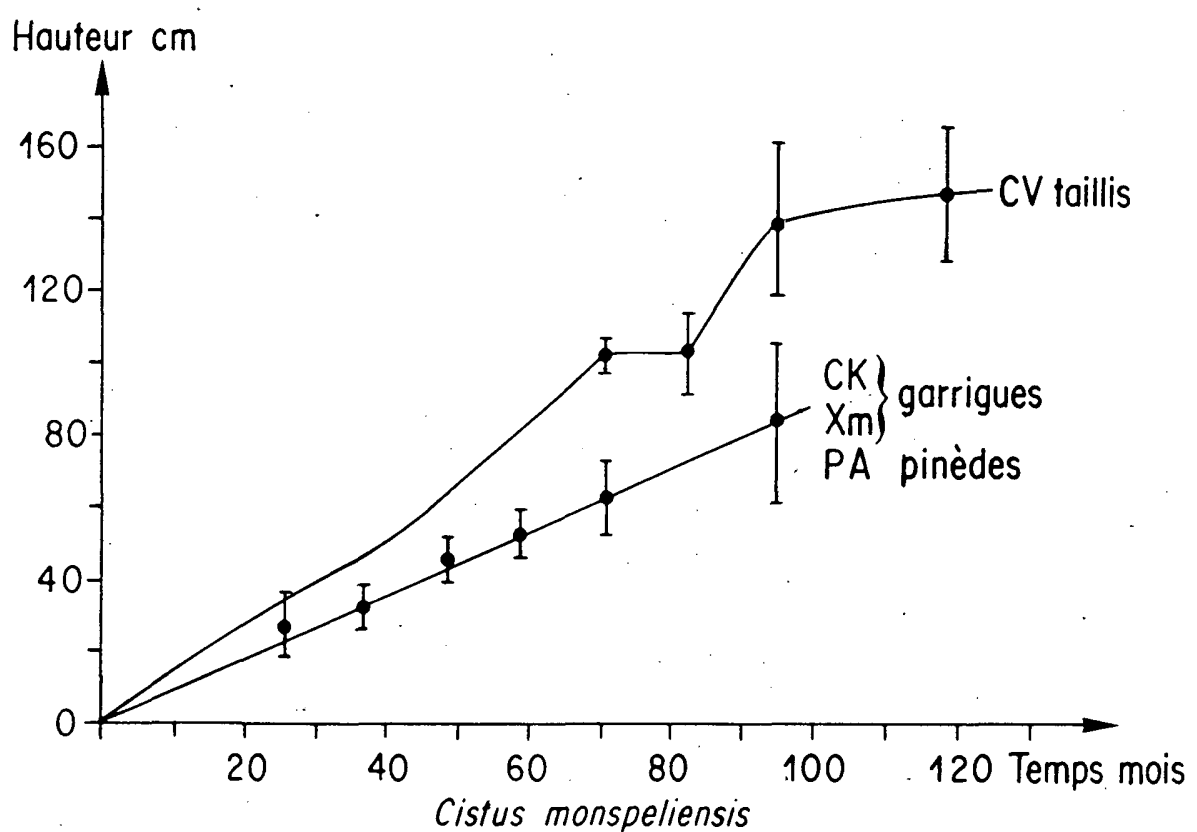

Fig. 8. Courbes de croissance des repousses de Cistus monspeliensis après incendie. Même légende que pour la figure 1. (Curvas de crecimiento de los rebrotes de Cistus monspeliensis tras incendio. Misma leyenda que para la fig. 1).

Rosmarinus officinalis, Q. coccifera, Q. ilex, seuls les deux Quercusprésentent des accroissements différents; ceux de Q. coccifera sont plus hauts, ceux de $Q$. ilex sont plus bas chez MANSANET TEROL: ces différences pourraient provenir du fait que cet auteur a observé des zones incendiées non forestières et dans une région (sud-est de l'Espagne) plus chaude et plus sèche que la région de Montpellier, donc plus favorable à $Q$. coccifera et plus défavorable à Q. ilex.

La plupart des espèces présentent des dimorphismes de vitesse de croissance. Lorsqu'elles sont dans des formations forestières leur croissance engendrerait plus rapidement des individus de haute taille que lorsque des individus de ces mêmes espèces vivent dans des garrigues. Cela peut être dû, dans les forêts, à une compétition pour la lumière; mais aussi au fait que les garrigues ont pu être parcourues beaucoup plus souvent par les incendies, et constituent des phytocénoses de taille basse.

Cette disparité de vitesses de croissance se manifestant entre individus d'une même espèce, mais vivant dans des milieux différents, ne semble pas, apparemment, avoir été décrite jusqu'à présent pour des rejets apparaissant après incendie. 
PIRINEOS 140

Deux influences semblent commander l'activité de croissance des repousses après incendie. La principale, liée génotype, tendrait à reconstituer la taille normale de l'adulte, caractéristique de l'espèce. L'autre, liée aux phénotypes, tiendrait compte de l'histoire (fréquences des feux) et de la structure des phytocénoses dans lesquelles vivent les individus.

\section{References}

Arianoutsou, M. (1984): Post-fire successional recovery of a phryganic (east Mediterranean) ecosystem. Acta Oecologica, Oecologica Plantarum, 5: 387-394.

Braun-Blanouet, J., Roússine, N. \& Negre, R. (1952): Les groupements végétaux de la France méditerranéenne. C.N.R.S., 297 p., Paris.

DEBUSSCHE, M. \& ISENMANN. P.(1983): La consommation de fruits chez quelques espèces de fauvettes méditerranéennes (Sylvia melanocephala, S. cantillans, S. hortensis, S. undata). Alauda, 51: 302-308.

Debussche, M., LePART, J. \& Molina, J. (1985): La dissémination des plantes à fruits charnus par les oiseaux: rôle de la structure de la végétation et impact sur la succession en région méditerranéenne. Acta Oecologica, Oecologica Generalis, 6: 65-80.

Emberger, L. (1942): Un projet de classification des climats au point de vue phytogéographique. Bulletin de Société Histoire Naturelle de Toulouse, 77: 97-124.

EMBERGER, L. (1971): Considèrations complémentaires au sujet des recherches bioclimatologiques et phytogéographiques-écologiques. In Travaux de Botanique et Ecologie, Masson, 291-301. Paris.

FowELLS, H. A. (1965): Silvics of forest trees of the United States. U.S.D.A. Agricultural Handbook, 271: 762 p., Washington.

GrifFIN, J. R. (1980): Sprouting in fire-damaged valley oaks, Chew Ridge, California. In Symposium on Ecology, Management and Utilization of California Oaks. U.S.D.A. General Technical Report PSW-44, 216-219, Pacific Southwest Forest and Rage Experiment Station.

MANSANET TEROL, C. M. (1987): Incendios forestales en Alicante. Estudio de la evolución de la vegetación quemada. Publicaciones Caja de Ahorros Provincial 143, 188 p., Alicante.

NAVEH, Z. (1974): The ecology of fire in Israel. In Tall Timbers Fire Ecology Conference, 13: $131-170$.

NAVEH, Z. (1975): The evolutionary significance of fire in the Mediterranean region. Vegetation, 29: 199-208.

PLumB, T. R. (1980): Reponse of oaks to fire. In Symposium on Ecology, Management and Utilization of California Oaks. U.S.D.A. General Technical Report PSW-44, 202215. Pacific Southwest Forest and Range Experiment Station.

ROTH, E. R. \& SLEETH, B. (1939): Butt rot in unburned sprout oak stands. U.S.D.A. Technical Bulletin, 684: 42 p., Washington.

TERRADAS, J. (coordinador) (1987): Ecosistemas terrestres. La resposta als incendis $i$ d'altres pertorbacions. Quaderns Ecologia Aplicada, 10: 205 p., Barcelona.

TrabaUd, L. (1970): Quelques valeurs et observations sur la phytodynamique des surfaces incendiées dans le Bas-Languedoc. Naturalia Monspeliensia, Série Botanique, 21: 231-242.

Trabaud, L. (1974): Experimental study of the effects of prescribed burning on a Quercus coccifera L. garrigue. In Tall Timbers Fire Ecology Conference 13: 97-129. 
REPONSES DES VEGETAUX LIGNEUX

TRABAUD, L. (1977): Comparison between the effect of prescribed fires and wild fires on the global quantitative development of the kermes scrub oak (Quercus cocciferaL.) garrigues. In Symposium on Environmental Consequences of Fire and Fuel Management in Mediterranean Ecosystems. U.S.D.A. General Technical Report WO-3, 272-283, Washington.

TrABAUD, L. (1980): Impact biologique et écologique des feux de végétation sur l'organisation, la structure et l'évolution de la végétation des garrigues du BasLanguedoc. Thèse Doctorat Etat Sciences, Université des Sciences et Techniques du Languedoc, 291 p., Montpellier.

TrABAUD, L. (1983): Evolution après incendie de la structure de quelques phytocénoses méditerranéennes du Bas-Languedoc (Sud de la France). Annales des Sciences Forestières, 40: 177-195.

TrABAUD, L. \& LEPART, J. (1980): Diversity and stability in garrigue ecosystems after fire. Vegetatio, 43: 49-57.

TRABAUD, L. \& LEPART, J. (1981): Floristic changes in a Quercus coccifera L. garrigue according to different fire regimes. Vegetatio, 46: 105-116.

Trabaud, L., Michels, C. \& Grosman, J. (1985): Recovery of burnt Pinus halepensis Mill. forests. II. Pine reconstitution after wildfire. Forest Ecology and Management, 13: 167-179. 